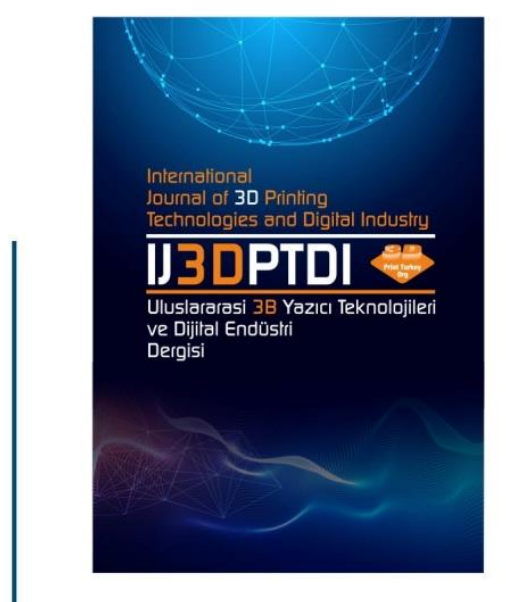

ULUSLARARASI 3B YAZICI TEKNOLOJILERI

VE DIJITAL ENDÜSTRI DERGISI

INTERNATIONAL JOURNAL QF 30 PRINTING TECHNOLOGIES AND DIGITAL INDUSTRY

IS5N:2602-3350 [Online]

URL: https://dergipark.org.tr/ij3dptdi

\title{
NON-PLANAR TOOLPATH FOR LARGE SCALE ADDITIVE MANUFACTURING
}

Yazarlar (Authors): Omer Eyercioglu ${ }^{D}$, Mehmet Aladag 10 *

Bu makaleye şu şekilde atıfta bulunabilirsiniz (To cite to this article): Eyercioglu O., Aladag M., "Non-Planar Toolpath For Large Scale Additive Manufacturing" Int. J. of 3D Printing Tech. Dig. Ind., 5(3): 477-487, (2021). 


\title{
NON-PLANAR TOOLPATH FOR LARGE SCALE ADDITIVE MANUFACTURING
}

\author{
Omer Eyercioglua (iD), Mehmet Aladaga (iD * \\ ${ }^{\mathrm{a}}$ Gaziantep Gaziantep University, Engineering Faculty, Mechanical Engineering Department, Gaziantep, TURKEY. \\ *Corresponding Author: mmehmetaladag@gmail.com
}

(Received: 23.06.2021; Revised: 06.08.2021; Accepted: 25.11.2021)

\begin{abstract}
The parts produced by additive manufacturing are inherently subjected to discretization effects due to their layer-based addition. The stair-stepping effect on the surface quality is inevitable for most of the techniques and it becomes more dominant for the regions having small surface inclinations. The stair-stepping influences the mechanical properties as well as the aesthetic perception. Many researchers have been presented several approaches to overcome or minimize the stair-stepping effects and improve the surface quality of additively manufactured parts. One of these methods is non-planar slicing. The stair-stepping effect was significantly decreased by this method. The attempts have been made generally for the FDMprinted objects, however, there is no or fewer efforts have been made for parts of large-scale additive manufacturing (LSAM). Due to higher deposition rates and larger nozzle diameters (i.e. bead size), the discretization effect is more in large-scale additive manufacturing. In this paper, the presented methods to mitigate the stair-stepping effect and improving the surface quality of additive manufacturing are reviewed, and practicing in large-scale 3D printing is discussed. Moreover, a preliminary experimental study of 3D printing with a non-planar toolpath was carried out and the results were presented.
\end{abstract}

Keywords: Large scale additive manufacturing (LSAM), non-planar 3D printing, stair-stepping effect, surface quality.

\section{INTRODUCTION}

Additive manufacturing (AM) is a manufacturing method that providing huge advantages, continuously develops for the future as well. It enables the manufacturability of complex parts thanks to the layered manufacturing (LM) methodology. Thereby, AM perhaps become the most popular among other manufacturing methods; an extruder moves linearly along three orthogonal axes to deposit melted material of a fused thermoplastic material onto the previous layer and, this progress continues according to manufactured geometry shape up to end [1].

Traditional AM process, the layers are sliced considering a slicing algorithm along the z-axis and parallel plane to the $x-y$ axis as flat layers by slicer software [2]. This is the main advantage of comparing AM with traditional subtraction manufacturing methods (i.e. milling). Also, even if a part has a simple geometry, selection of tools, and creating suitable tool paths can be simple, however, for complex parts, manufacturing becomes difficult or sometimes impossible as well. Therefore, LM methodology can be the first choice in the manufacturing of complex parts obviously. However, produced parts by AM are not efficient completely; besides mechanical properties, geometrically aesthetic appearance has importance. In the traditional AM process, the model is sliced layer by layer as planar in order to create a tool path, and the model is manufactured with $2.5 \mathrm{D}$ axes [3]-[5]. Considering if there is a curvature surface that will be manufactured additively, some stair-step effects are observed that significantly affects surface quality. Because stair-step effects are seen further in inclined or rounded (non-planar) surfaces. This directly affects the aesthetic appearance. But, stair-step effect not only affects the aesthetic appearance but can also influence the mechanical properties, e.g., the aerodynamic performance [6]-[10]. Also, planar slicing affects the surface roughness negatively [11]. So, printed with planar layer is not satisfy totally. In order to decrease the stair-step effect, many studies were made; some researchers were made post-processing operations (i.e. milling, smoothing, painting, and so on) after the model printed, hence, the surface quality 
is increased but, the size of geometry obviously changed as it expected. Some of the researchers studied the slicing methodology [12]-[15]; layer thicknesses were changed to eliminate or decrease the stair-step effect, this is called adaptive slicing [16]. However, this approach has not been become satisfied totally. Starting from this point, a new slicing method was presented by engineers; non-planar 3D printing (another word; non-flat layer, variable layer, or active $\mathrm{Z}$ printing) has been proposed to improve the printing quality [17]. Many studies have been carried out on non-planar 3D printing. Before mentioning studies, non-planar printing was proposed firstly by Chakraborty [18]. Regarding the surface quality, non-planar printing lead to a decrease in the stair-step effect significantly [19]. Zhao and Ding [20], [21], stair-step effect can be reduced by generating multi-directional curved layer 3D printing. The curved layer supports active $\mathrm{z}$ printing tool path trajectory.

Many studies were made to create a curved tool path [22]-[25]. Chakraborty et al. [18] introduced this algorithm to generate a curved layer toolpath. In their work, each surface is defined as a parametric surface, the tool path is generated onward these surfaces; they also considered the printability of the curved surfaces; thus, the algorithm is divided into two sections; proper toolpath generation and extrusion orientation. However, this algorithm was only presented, it was not tried experimentally on an object. Another researcher; Huang et al. tried this algorithm with different parameters experimentally besides electronically and mechanically, and their studies are still ongoing [26]. Llewellyn-Jones et al. proposed three case studies demonstrating the ability of the $\mathrm{G}$ code generation algorithm to solve concave and convex structures were shown. Experimental results have clearly demonstrated the effectiveness of using curved layers to improve the surface finish of a printed component [27]-[29]. The case study examples used in their study were specifically selected to demonstrate the effectiveness of the slicing algorithm and consequently not suitable for mechanical testing. Consequently, the printing time in non-planar printing was seen that longer than comparing of planar layer printing [6], [20]. There is a report that mentions how to decrease the stairstepping effect [12]. The stair-stepping effect can be decreased by decreasing the sliced layer thickness. However, as decreasing the layer means longer printing time, this not recommend due to the repercussion of the high cost [30]-[33]. Developing the technology, the availability of material and printing system technology was relatively enhanced. Therefore, even if adaptive slicing causes more printing time, it was seen that the software can decrease time relatively. Ahlers et al. developed an algorithm on open-source slicing software (Slic3r). In their software, besides non-planar surface tool path generation, an adaptive slicing algorithm was developed. Thanks to this algorithm, decreased printing time as well as geometrical aesthetic appearance, was enhanced [6,34-35].

All the work done so far, the attempts had been made generally for the FDM-printed objects, however, there is no or fewer efforts had been made for parts of large-scale additive manufacturing (LSAM). Due to higher deposition rates (up to $50 \mathrm{~kg} / \mathrm{hr}$ ) and larger nozzle diameters (i.e., bead size), the discretization effect is more in LSAM [36]. In this study, the methods presented to reduce the stair-step effect of layered manufacturing and improve the surface quality are reviewed and the application in large-scale 3D printing is discussed. A preliminary experimental study of 3D printing with a non-planar tool path was performed and the results were presented.

\section{DEVELOPED SLICING ALGORITHMS TO DECREASE STAIR-STEPPING EFFECT 2.1 Definition of Stair-Stepping Effect}

A model is sliced layer by layer parallel to the $x-y$ plane by software, and the appearance of the geometry seems layer by layer. In this situation, if a geometry has an inclined or curved surface, the edge of the geometry is seen as a ladder (See in Figure 1). Therefore, it is called as stair-step effect. Many spare parts have been started to produce using FDM recently. In particular, for old parts which are not suitable for mass production, AM is an invaluable technology. Because AM does not require special tools such as mold, cutting tools, etc. But additively manufactured parts cannot be compared completely with other conventionally manufactured parts. In that considered geometrical aesthetic appearance and mechanical characteristics, AM stays behind. Even so, researchers continuously studying to enhanced additively manufactured parts in terms of characteristics. As a result of those researches, two methods are in the foreground; Adaptive slicing method, non-planar toolpath generating. The methodologies were described in detail in the following of this article. 


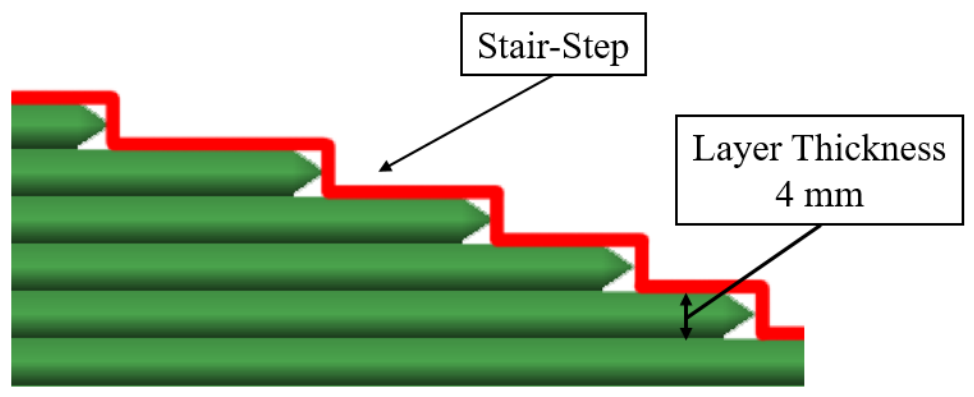

Figure 1. Stair-Stepping in a curvature top surface.

\subsubsection{Adaptive Slicing}

Adaptive slicing is a slicing feature that always used by sliced software [37]. This is a parameter that can be optimized by the slicer software' user interface and, it is activated default. Adaptive slicing uses an algorithm; especially in rounded or non-planar surfaces automatically changes the layer thicknesses. Layer thickness is reduced in the non-uniform surface in order to decrease the stair-step effect, this is the methodology as a matter of fact. An example was given in Figure 1. In this figure, two sliced models were given; one is sliced straight, second is sliced using an adaptive slicing algorithm. When this 3D model divided into two-part from a horizontal dashed line, in the part below layers are seen totally planar. Adaptive slicing will not configure layer thickness due to the flat geometry, however, geometry is not flat considering the top surface in the part above. The adaptive slicing algorithm will operate automatically here.

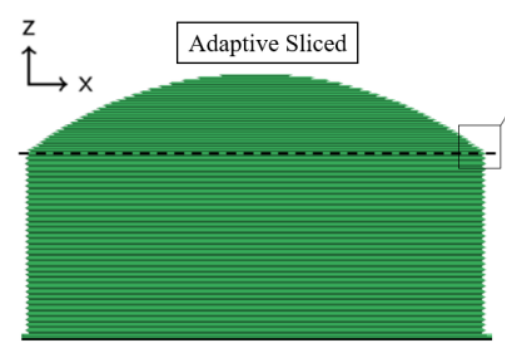

a
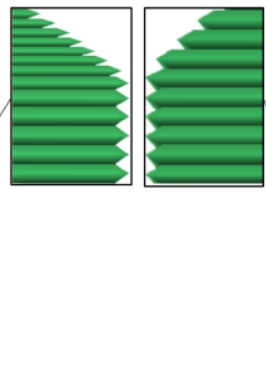

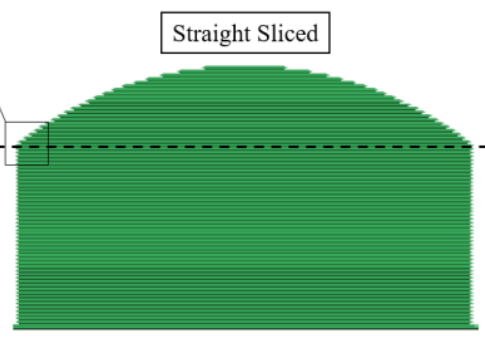

b

Figure 2. Comparison of adaptive slicing (a) and straight (constant layer thickness) slicing (b).

\subsubsection{Non-Planar Slicing}

Most common slicer tools slice model parallel to the X-Y plane. The tool path is $2.5 \mathrm{D}$ because of planar layers. So, the Model is produced by a $2.5 \mathrm{D}$ axis toolpath. In non-planar slices, the tool path fully 3D because of non-planar slicing, therefore, all axes fully actively work during printing. Non-planar slicing is divided into two groups; one of them only considers the top surface and, the non-planar tool path is generated only for the top surface [37], [38]. An example was given in figure 3. In the top layer non-planar slicing, the tool path is generated $2.5 \mathrm{D}$ from the previous top layer. Then, a fully $3 \mathrm{D}$ toolpath is generated by the software; the extruder moves by following top layer surface geometry.
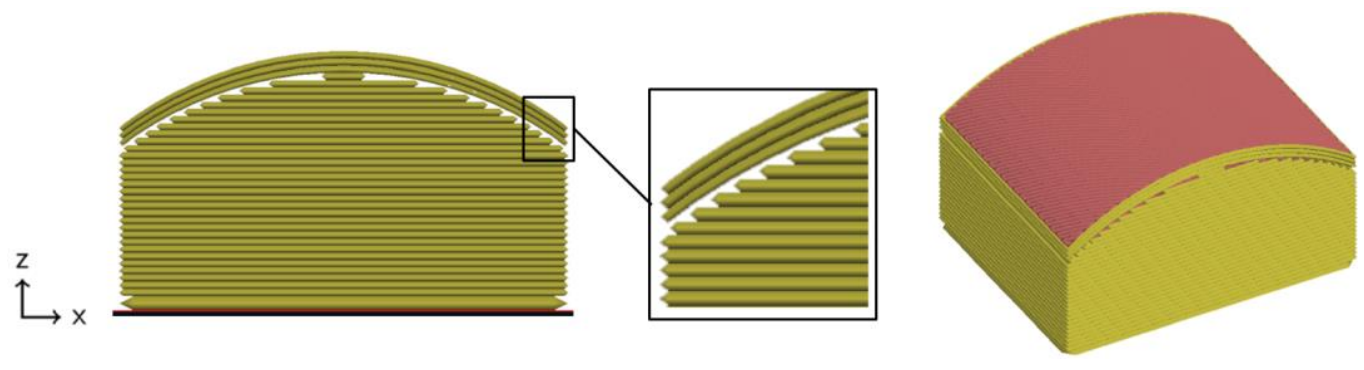

Figure 3. Non-Planar top-layer slicing. 
The second one is whole non-planar slicing in which a non-planar toolpath is generated from the first sliced layer to the last layer (top surface). This is also called active $\mathrm{Z}$ printing or variable depth curved layer; layers are not planar (See Figure 4) [1], [3]. In this slicing method, the extruder is in motion in z-direction according to the top layer geometry. While the extruder moving in the z-direction, layer thickness changes and the material flow rate relatively changes at the same time. Because changing the layer thickness changes the layer volume as well. In order to fill the required layer volume, the extruder increases or decreases the deposition rate.

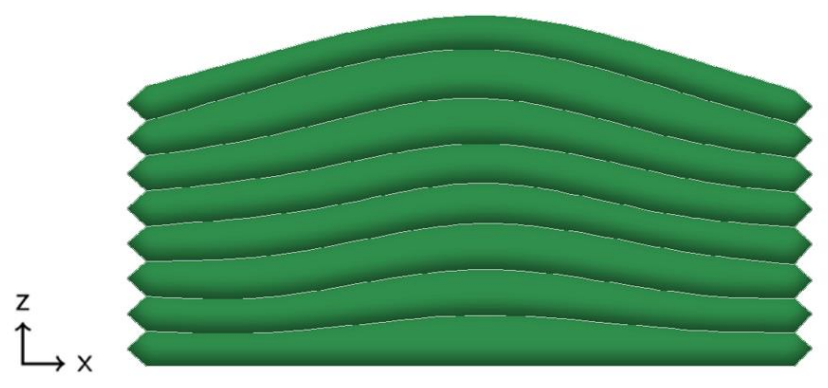

Figure 4. Non Planar whole layer slicing.

\subsection{Limitations of Non-planar Slicing}

Both in non-planar and planar slicing obviously, the appearance of the vertical wall of the part will be layer by layer in LSAM. That's why produced part is subjected to post-processing operation. This is generally in the natural process for parts which was produced by LSAM. Considering this, non-planar printing also provides advantages in every way. However, there are some mechanical limitations in non-planar printing; these are collisions and nozzle orientation to layer in angular surfaces [5], [6]. Due to the geometry of the extruder and nozzle, it may not be possible to predict the collisions by software. Therefore, while toolpath generation for non-planar surfaces, amount of surface slope of the geometry should be regarded. Figure 5 presents LSAM direct extrusion nozzle and its collision area.

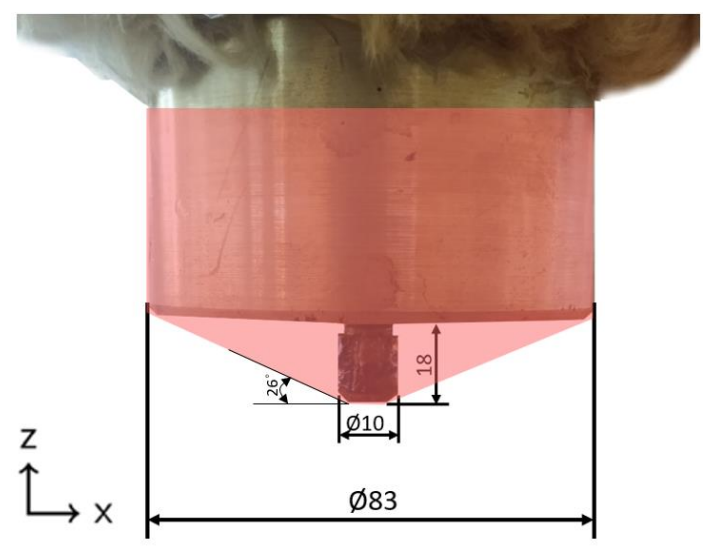

Figure 5. Direct extrusion system of LSAM and its collision area (dimensions are in mm).

A similar study on standard small-scale commercial 3D printers was made and, collision detection algorithms were presented. This algorithm needs some parameters such as nozzle outer diameter, nozzle height, and minimum and maximum non-planar collision angle. Then the model is sliced and a non-planar 
tool path generated. In accordance with these given parameters, software limits non-planar tool path generation even if the surface is fully non-planar (See Figure 6).
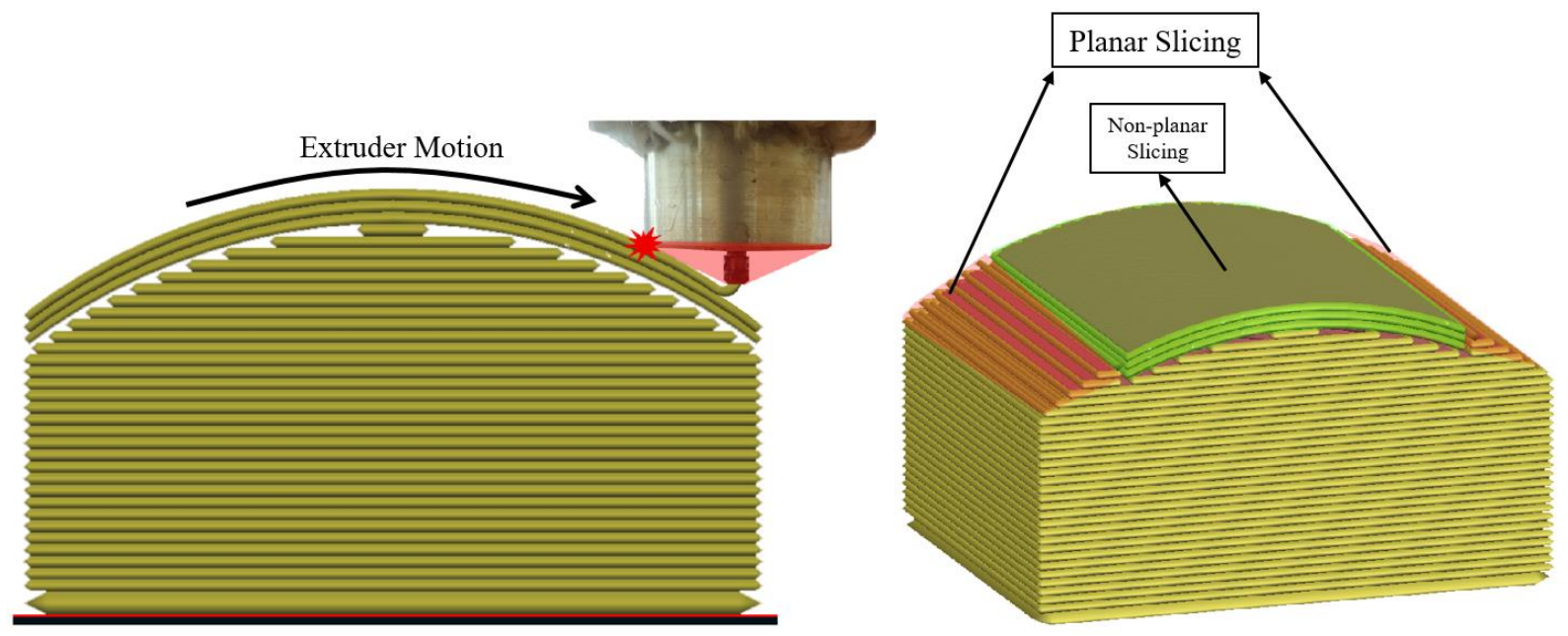

Figure 6. Model is sliced partially planar and partially non-planar due to the collision parameters and, collision.

\section{EXPERIMENTAL STUDY}

\subsection{Material}

An Acrylonitrile Butadiene Styrene (ABS) thermoplastic polymer, with the properties shown in Table 1 was used in the experimental study. The granules were dried at $80^{\circ} \mathrm{C}$ for 4 hours before use. The material is deposited at $240^{\circ} \mathrm{C}$ onto a heated building plate at $80^{\circ} \mathrm{C}$.

Table 1. The mechanical properties of ABS.

\begin{tabular}{lll}
\hline \hline Properties & Unit & Value \\
\hline \hline Density & $\mathrm{kg} / \mathrm{m}^{3}$ & 1060 \\
Thermal Conductivity & $\mathrm{K}(\mathrm{W} / \mathrm{mK})$ & 0.177 \\
Specific Heat & $\mathrm{C}(\mathrm{J} / \mathrm{KgK})$ & 2080 \\
Emissivity & $\varepsilon$ & 0.87 \\
Glass Transition Temperature & $\mathrm{T}_{\mathrm{g}}\left({ }^{\circ} \mathrm{C}\right)$ & 105 \\
\hline \hline
\end{tabular}

\subsection{LSAM System}

A direct extrusion system shown in Figure 7 is designed, manufactured and it was replaced with the spindle of the 3-axes $\mathrm{CNC}$ unit available in the department. The maximum displacements in $\mathrm{X}, \mathrm{Y}$, and $\mathrm{Z}$ directions are 1800, 2500, and $400 \mathrm{~mm}$, respectively. The extruder is a single screw extruder and it is driven by a variable speed motor. The ABS granules are feeding through the extruder by an automatic feeder. The feed rate of granules and the speed of the screw can be controlled to melt and deposit molten polymer at a rate consistent with the movement of the axes (building speed) and desired bead profile. The barrel has band heaters and a control unit to keep the chamber and nozzle temperatures in the required ranges. Also, to keep the environment temperature, the printing area was covered, even, a heated bed was placed inside of printing system. Thus, the environment temperature was kept steady. Thereby, the amount of deflection caused by fast cooling was decreased. 


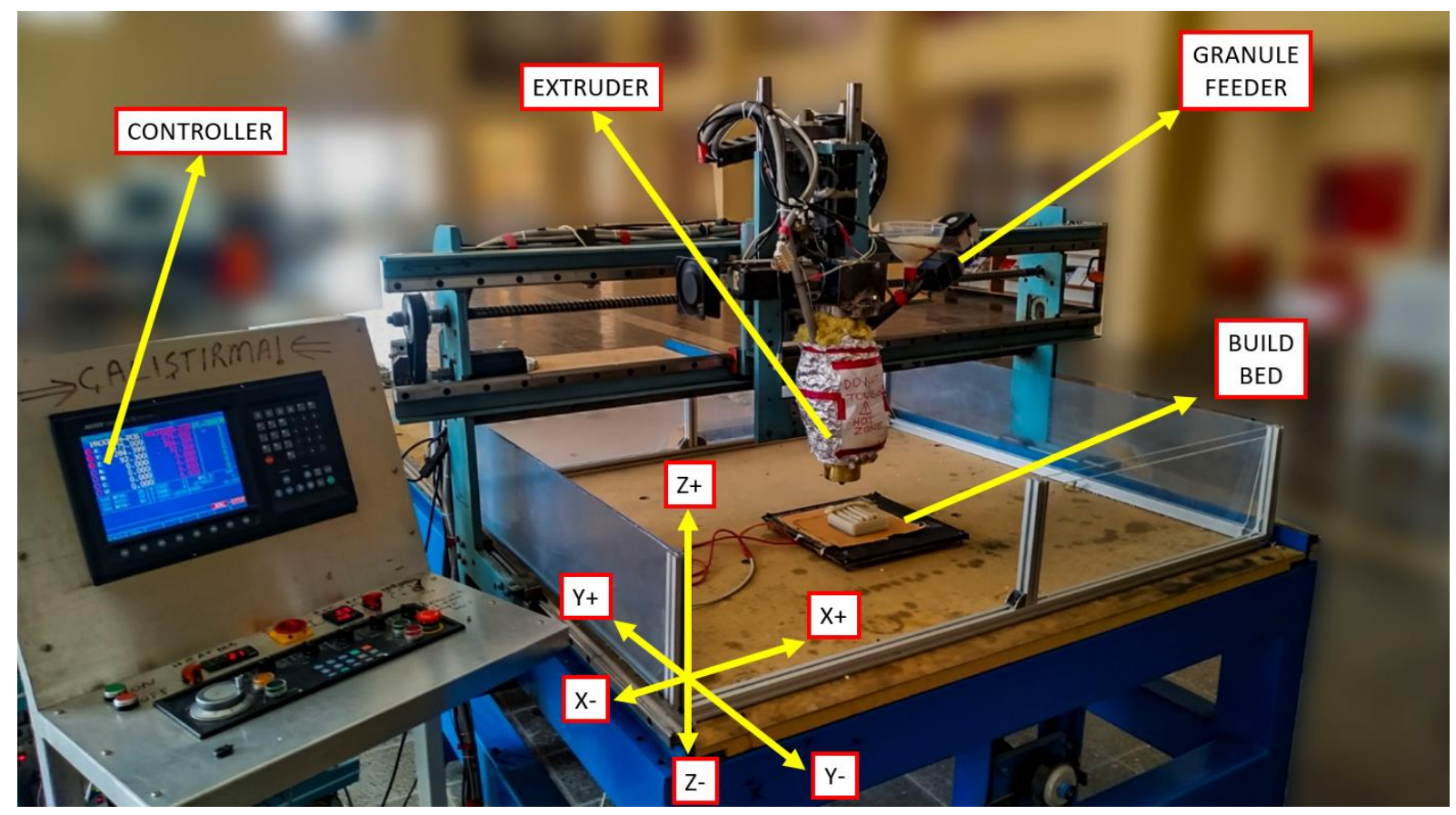

Figure 7. LSAM system in mechanical engineering laboratory, Gaziantep University.

\subsection{Modeling and Slicing}

A non-planar geometry on the upper surface is designed. The reason for this is to see that the ladder-step effect is eliminated by generating a 3D toolpath on a non-planar surface. In addition, the distance of the inner fill lines from each other also has an effect on the geometry of the non-planar surface. Therefore, many findings can be reached on this model designed in the experimental study. This solid model was drawn by using Solidworks in order to print in LSAM. Next, model transferred to the slicer software in order to slice non-planar (See Figure 8). The printing parameters (nozzle diameter, layer thickness, printing temperature, printing speed) were defined as well. The non-planar slicing parameters were determined according to our LSAM machine system. These parameters and values were given in table 2 .
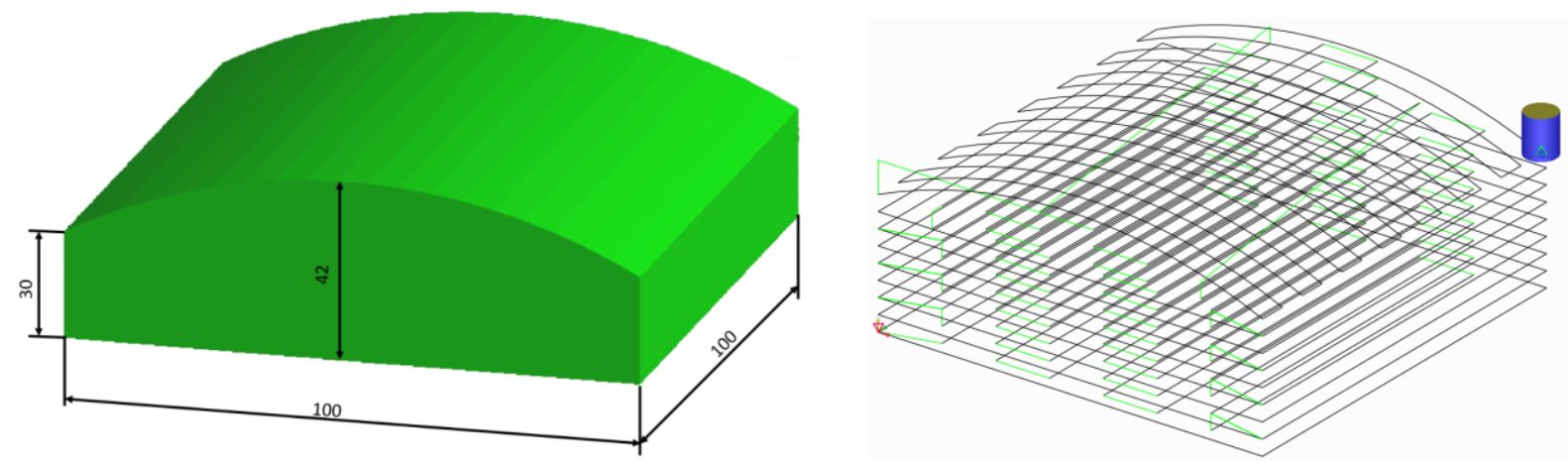

Figure 8. 3D model with dimensions and non-planar toolpath.

Table 2. Non-planar slicing parameter 


\begin{tabular}{|c|c|c|}
\hline Parameter & Unit & Value \\
\hline Nozzle Diameter & $\mathrm{mm}$ & 6 \\
\hline Deposition Temperature & ${ }^{\circ} \mathrm{C}$ & 240 \\
\hline Layer Height & $\mathrm{mm}$ & 4 \\
\hline Printing Speed & $\mathrm{mm} / \mathrm{min}$ & 400 \\
\hline Infill type & - & Line \\
\hline Adaptive Slicing & - & Activated \\
\hline Adaptive Quality & $\%$ & 75 \\
\hline \multicolumn{3}{|c|}{ For Non-planar Layers } \\
\hline Maximum non-planar angle & degree & 26 \\
\hline Maximum non-planar collision angle & degree & 26 \\
\hline Minimum non-planar area & $\mathrm{mm}^{2}$ & 80 \\
\hline Maximum non-planar collision height & $\mathrm{mm}$ & 18 \\
\hline Ignore collision size & $\mathrm{mm}^{2}$ & 5 \\
\hline
\end{tabular}

\subsection{Printing}

Generated non-planar toolpath for producing the desired model was operated and printed with our LSAM machine. The printing parameters and toolpath were already obtained in the previous steps. The extrusion temperature and bed temperature were set at $240{ }^{\circ} \mathrm{C}$ and $80{ }^{\circ} \mathrm{C}$, respectively. The printing parameters were already obtained in our previous studies. These parameters and values were given in table 3 . An image was given during the printing with LSAM in Figure 9.

Table 3. LSAM printing parameters

\begin{tabular}{lll}
\hline Parameter & Unit & Value \\
\hline \hline Nozzle Diameter & $\mathrm{mm}$ & 6 \\
Deposition Temperature & ${ }^{\circ} \mathrm{C}$ & 240 \\
Bed Temperature & ${ }^{\circ} \mathrm{C}$ & 80 \\
Printing Speed & $\mathrm{mm} / \mathrm{min}$ & 400 \\
\hline \hline
\end{tabular}
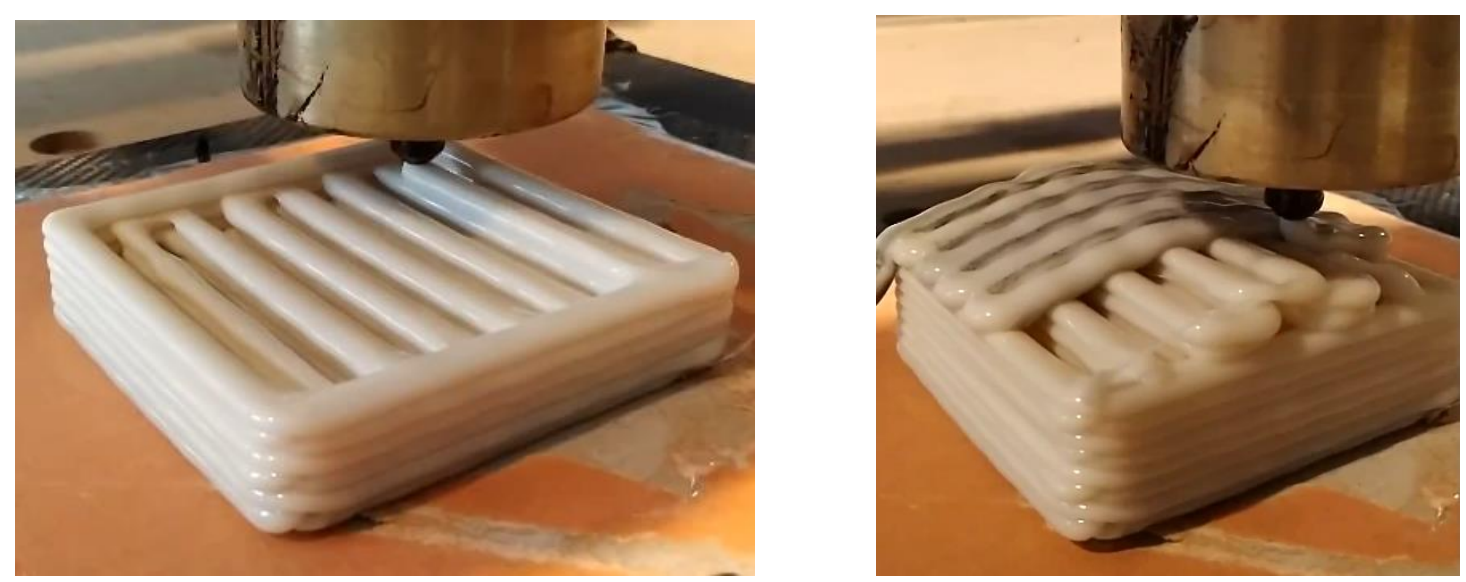

Figure 9. Printing of the part with LSAM. 


\section{RESULT AND DISCUSSION}

Produced part with LSAM was shown in Figure 10. Because of the high layer thickness and nozzle, the layers on the wall are obviously seen. The wall surface of the part that was produced with LSAM was machined with a milling operation (See Figure 10). The purpose of machining the wall is to see any gap between layers. Also, under the non-planar material deposited on the top surface layer, planar sliced layers are there. And, these layers seem like stair-step. Because of the stair-step effect in the planar layers, some gaps took place as the material was deposited and passing from layer to layer (see Figure 11). We can call this "error". Thus, occurred errors can be seen easily after the milling operation.

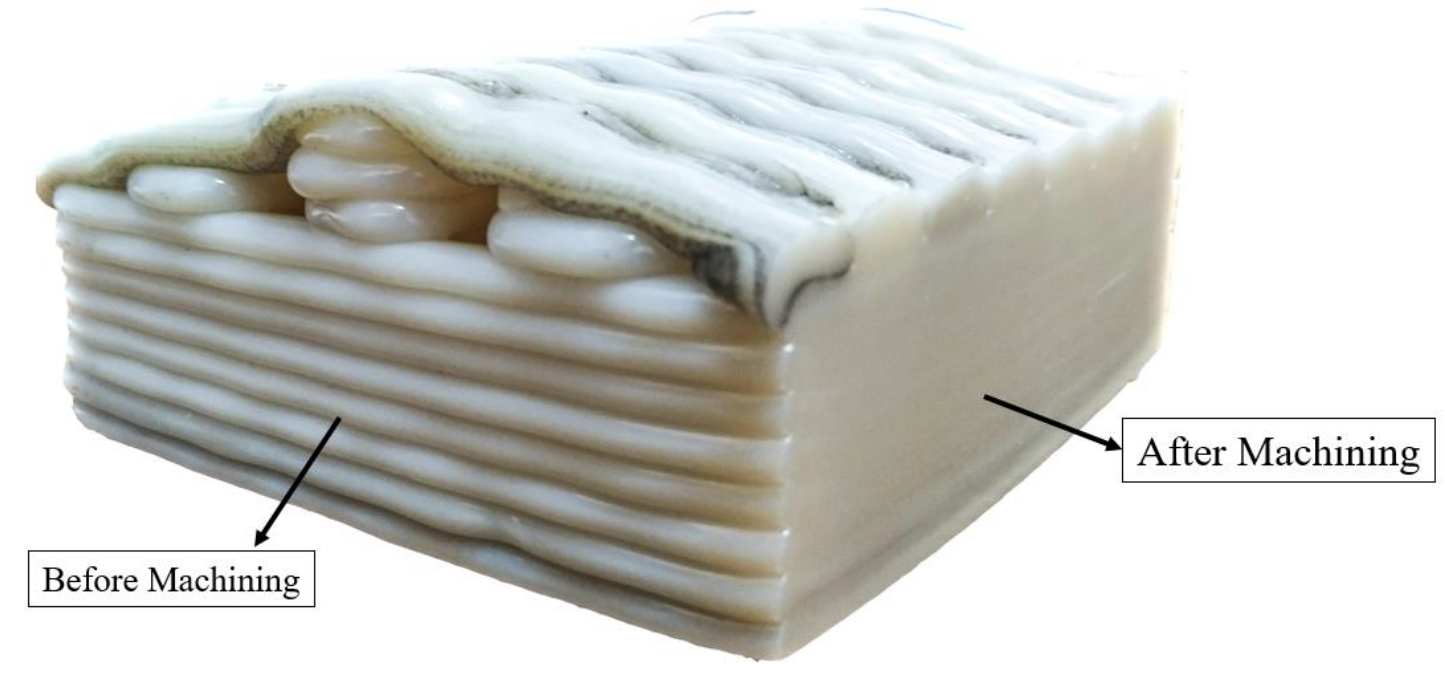

Figure 10. Wall surface conditions before and after machining.

Moreover, some amount of saggings were observed passing from one layer to another layer (see Figure 11). The reason for this is the maximum bridging capacity of the single bead. In our previous study, we investigated the maximum bridging distance and the suitable bridging length was $6.36 \mathrm{~mm}$ without air cooling the bead, $11.18 \mathrm{~mm}$ with air cooling the bead [39]. So, the saggings were due to the bridging distance. In our printed model, the spaces between infill lines were greater than $6.36 \mathrm{~mm}$.
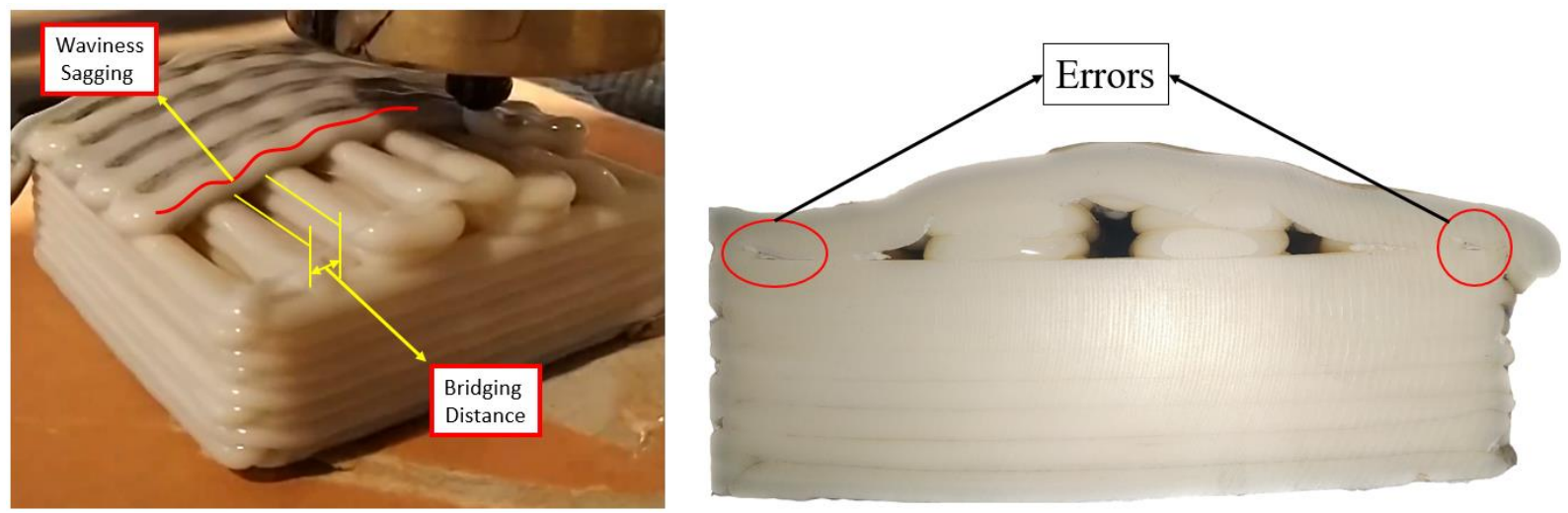

Figure 11. Saggings due to the wide infill lines (in the left) and air gap (error) (in the right).

\section{CONCLUSION}

As a consequence the followings can be concluded from experimental study;

Even though planar slicing is most commonly used in LM, considering the final product, hence, the planar sliced model has advantages in terms of production time as well. Even if the adaptive slicing method providing advantages in order to decrease the stair-step effect, it obviously increases the production time [40]. But some limitations are there in non-planar printing such as collision and nozzle orientation. These 
limitations can be removed by redesigning the extruder nozzle geometry, even adding an axis to gain motion flexibility [3], [41]. When viewing from this aspect, the planar slicing stays behind non-planar slicing in terms of surface quality and mechanical property. Open-source slicing software is adopted to LSAM using a previously developed non-planar algorithm. The non-planar slicing was therefore enabled. The stair-step appearance totally covered in the top surfaces by a non-planar toolpath. Thereby, the surface quality and aesthetic appearance significantly enhanced on the non-planar top surface [42].

Even if the top surface non-planar printed, some waviness was observed due to the long bridging of the bead. In our printed model, the spaces between infill lines must have been a maximum of $6.36 \mathrm{~mm}$. So, the surface waviness is eliminated or, forced air cooling can be conducted or, two superimposed non-planar layers can be deposited. In addition to this, some errors were seen sourced by the planar layer and stair-step effect under the top non-planar surface. These errors caused by the gaps may decrease the density of the non-planar top surface. Even if we suppose the bridging distance is well enough, the errors are not totally eliminated. Because the internal corner will have an air gap [34]. And, it brings along a weakness in terms of mechanical characteristics and aesthetic appearance. A slicing software-based solution can be recommended; an algorithm can be developed to fill that mentioned air gaps by increasing the material deposition rate.

The study on this subject continues within the scope of a thesis. The outputs obtained in this article are associated with production parameters (toolpath optimization, layer thickness, printing speed, nozzle orientation) and experimental studies continue.

\section{ACKNOWLEDGMENT}

The authors would like to acknowledge the contributions of the Scientific Project Bureau (BAPYB) of The Gaziantep University.

\section{REFERENCES}

1. Chen L., Chung M.F., Tian Y., Joneja A., Tang K., "Variable-depth curved layer fused deposition modeling of thin-shells", Robotics and Computer-Integrated Manufacturing, Vol. 57, Issue January, 2019.

2. Mohan Pandey P., Venkata Reddy N., Dhande S.G., "Slicing procedures in layered manufacturing: A review", Rapid Prototyping Journal, Vol. 9, Issue 5, 2003.

3. Pelzer L., Hopmann C., "Additive manufacturing of non-planar layers with variable layer height", Additive Manufacturing, Vol. 37, Issue May 2020, 2021.

4. Gosselin C., Duballet R., Roux P., Gaudillière N., Dirrenberger J., Morel P., "Large-scale 3D printing of ultrahigh performance concrete - a new processing route for architects and builders", Materials and Design, Vol. 100, 2016.

5. Micali M.K., Dornfeld D., "Fully three-dimensional toolpath generation for point-based additive manufacturing systems", Solid Freeform Fabrication 2016: Proceedings of the 27th Annual International Solid Freeform Fabrication Symposium - An Additive Manufacturing Conference, SFF 2016, 2016.

6. Ahlers D., "3D printing of nonplanar layers for smooth surface generation", University of Hamburg, 2018.

7. Allum J., Kitzinger J., Li Y., Silberschmidt V. V., Gleadall A., "ZigZagZ: Improving mechanical performance in extrusion additive manufacturing by nonplanar toolpaths", Additive Manufacturing, Vol. 38, Issue November 2020, 2021.

8. Xu J., Sheng H., Zhang S., Tan J., Deng J., "Surface accuracy optimization of mechanical parts with multiple circular holes for additive manufacturing based on triangular fuzzy number", Frontiers of Mechanical Engineering, 2021.

9. Khurana J.B., Dinda S., Simpson T.W., "Active - Z printing: A new approach to increasing3D printed part strength", Solid Freeform Fabrication 2017: Proceedings of the 28th Annual International Solid Freeform Fabrication 
Symposium - An Additive Manufacturing Conference, SFF 2017, 2020.

10.Es-Said O.S., Foyos J., Noorani R., Mendelson M., Marloth R., Pregger B.A., "Effect of layer orientation on mechanical properties of rapid prototyped samples", Materials and Manufacturing Processes, Vol. 15, Issue 1, 2000.

11. Feng X., Cui B., Liu Y., Li L., Shi X., Zhang X., "Curved-layered material extrusion modeling for thin-walled parts by a 5-axis machine", Rapid Prototyping Journal, Vol. 27, Issue 7, 2021.

12. Tyberg J., Bohn J.H., "Local adaptive slicing for Layered Manufacturing", Rapid Prototyping Journal, Vol. 4, Issue 3, 1998

13. Sabourin E., Houser S.A., Bøhn J.H., "Adaptive slicing using stepwise uniform refinement", Rapid Prototyping Journal, Vol. 2, Issue 4, 1996.

14.Zhou M.Y., Xi J.T., Yan J.Q., "Adaptive direct slicing with non-uniform cusp heights for rapid prototyping", International Journal of Advanced Manufacturing Technology, Vol. 23, Issue 1-2, 2004.

15. Cormier D., Unnanon K., Sanii E., "Specifying non-uniform cusp heights as a potential aid for adaptive slicing", Rapid Prototyping Journal, Vol. 6, Issue 3, 2000.

16. Huang B., Singamneni S., "A mixed-layer approach combining both flat and curved layer slicing for fused deposition modelling", Proceedings of the Institution of Mechanical Engineers, Part B: Journal of Engineering Manufacture, Vol. 229, Issue 12, 2015.

17. Mohan Pandey P., Venkata Reddy N., Dhande S.G., "Slicing procedures in layered manufacturing: A review", Rapid Prototyping Journal, Vol. 9, Issue 5, 2003.

18. Chakraborty D., Aneesh Reddy B., Roy Choudhury A., "Extruder path generation for Curved Layer Fused Deposition Modeling", CAD Computer Aided Design, Vol. 40, Issue 2, 2008.

19. Cendrero A.M., Fortunato G.M., Munoz-Guijosa J.M., De Maria C., Díaz Lantada A., "Benefits of Non-Planar Printing Strategies Towards Eco-Efficient 3D Printing", Sustainability, Vol. 13, Issue 4, 2021.

20. Zhao H. ming, He Y., Fu J. zhong, Qiu J. jiang, "Inclined layer printing for fused deposition modeling without assisted supporting structure", Robotics and Computer-Integrated Manufacturing, Vol. 51, 2018.

21. D. D., Pan Z., D. Cuiuri H. L., N. L., S. van D., "Multi-Direction Slicing of STL Models for Robotic Wire-Feed Additive Manufacturing", in International Solid Freeform Fabrication Symposium, Pages 1059-1069, 2015.

22. Llewellyn-Jones T., Allen R., Trask R., "Curved Layer Fused Filament Fabrication Using Automated Toolpath Generation", 3D Printing and Additive Manufacturing, Vol. 3, Issue 4, 2016.

23. Diegel O., Singamneni S., Huang B., Gibson I., "Getting rid of the wires: Curved layer fused deposition modeling in conductive polymer additive manufacturing", Key Engineering Materials, Vol. 467-469, 2011.

24. Singamneni S., Roychoudhury A., Diegel O., Huang B., "Modeling and evaluation of curved layer fused deposition", Journal of Materials Processing Technology, Vol. 212, Issue 1, 2012.

25. Huang B., Singamneni S., "Curved layer fused deposition modeling with varying raster orientations", Applied Mechanics and Materials, Vol. 446-447, 2014.

26. Huang B., Singamneni S., Diegel O., "Construction of a curved layer rapid prototyping system: Integrating mechanical, electronic and software engineering", 15th International Conference on Mechatronics and Machine Vision in Practice, M2VIP'08, 2008.

27. Shembekar A. V., Yoon Y.J., Kanyuck A., Gupta S.K., "Trajectory planning for conformal 3D printing using nonplanar layers", Proceedings of the ASME Design Engineering Technical Conference, Vol. 1A-2018, 2018.

28. Allen R.J.A., Trask R.S., "An experimental demonstration of effective Curved Layer Fused Filament Fabrication utilising a parallel deposition robot", Additive Manufacturing, Vol. 8, 2015. 
29. Jin Y., Du J., He Y., Fu G., "Modeling and process planning for curved layer fused deposition", International Journal of Advanced Manufacturing Technology, Vol. 91, Issue 1-4, 2017.

30. De Jager P.J., Broek J.J., Vergeest J.S.M., "Using adaptive ruled layers for Rapid Prototyping: principles and first results", Solid Freeform Fabrication Symposium Proceedings, 1996.

31. Hope R.L., Roth R.N., Jacobs P.A., "Adaptive slicing with sloping layer surfaces", Rapid Prototyping Journal, Vol. 3, Issue 3, 1997.

32. Hope R.L., Jacobs P.A., Roth R.N., "Rapid prototyping with sloping surfaces", Rapid Prototyping Journal, Vol. 3, Issue 1, 1997.

33. Thomas C.L., Gaffney T.M., Kaza S., Lee C.H., "Rapid prototyping of large scale aerospace structures", in IEEE Aerospace Applications Conference Proceedings, Vol. 4, Pages 219-229, 1996.

34. Ahlers D., Wasserfall F., Hendrich N., Zhang J., "3D printing of nonplanar layers for smooth surface generation", IEEE International Conference on Automation Science and Engineering, Vol. 2019-Augus, Issue September, 2019.

35. Liu S., Joneja A., Tang K., "A new adaptive slicing algorithm based on slice contour reconstruction in layered manufacturing process", Computer-Aided Design and Applications, Vol. 18, Issue 6, 2021.

36. Eyercioglu O., Aladag M., Sever S., "Temperature Evaluation and Bounding Quality of Large Scale Additive Manufacturing Thin Wall Parts", Sigma J Eng \& Nat Sci, Vol. 36, Issue 3, 2018.

37. "Slic3r - Open source 3D printing toolbox". https://slic3r.org/, April 7, 2021.

38. "GitHub - DrEricEbert/Slic3r_NonPlanar_Slicing: Slic3r with integrated electronics and Nonplanar slicer (see branches)". https://github.com/DrEricEbert/Slic3r_NonPlanar_Slicing, April 7, 2021.

39. Eyercioglu O., Aladag M., Aksoy A., Gov K., "Determination of The Maximum Bridging Distance in Large Scale Additive Manufacturing", in 4th International Congress on 3d Printing (Additive Manufacturing) Technologies and Digital Industry, Pages 40-48, 2019.

40. Zhao G., Ma G., Feng J., Xiao W., "Nonplanar slicing and path generation methods for robotic additive manufacturing", International Journal of Advanced Manufacturing Technology, Vol. 96, Issue 9-12, 2018.

41. Xie F., Chen L., Li Z., Tang K., "Path smoothing and feed rate planning for robotic curved layer additive manufacturing", Robotics and Computer-Integrated Manufacturing, Vol. 65, 2020.

42. Shan Y., Gan D., Mao H., "Curved Layer Slicing based on Isothermal Surface", Procedia Manufacturing, Vol. 53, 2021. 